\title{
Fountain of Youth: aged blood-forming stem cells could be rejuvenated by young microenvironment
}

\author{
Tong Yin ${ }^{1}$, Linheng $\mathrm{Li}^{1}$ \\ ${ }^{1}$ Stowers Institute for Medical Research, 1000 E. $50^{\text {th }}$ St, Kansas City, MO 64110, USA \\ Cell Research (2010) 20:504-505. doi:10.1038/cr.2010.60; published online 3 May 2010
}

A recent paper published in Nature by Amy J Wagers' group reports a remarkable function of osteoblastic niche (defined as microenvironment) [1] in reversing the aged phenotype of hematopoietic (blood-forming) stem cells, thus opening the possibility for clinical treatment of age-related diseases via modifying the stem cell niche.

Aging of hematopoietic stem cells (HSCs) leads to gradual decline of regenerative potential, deficiency in immune function, loss of homeostatic control, and increased incidence of malignant diseases such as leukemia. Previous studies have mainly focused on understanding the intrinsic factors that influence aging of adult stem cells. For example, it has been shown that change in intrinsic factors, such as DNA damage, oxidative stress etc., is associated with HSC aging [2, 3]. Using in vivo imaging with multiphoton intravital microscopy, researchers observed that aged hematopoietic stem progenitor cells (HSPCs) changed locations, moving farther away from the endosteum (where quiescent HSCs normally reside) than did young HSPCs [4], which suggests extrinsic signals also play a role in HSC aging. However, it is not clear how the HSC niche influences HSC aging.

The HSC niche was first proposed in 1978 by Schofield to describe the

Correspondence: Linheng Li

E-mail: 1il@stowers-institute.org physiologically limited microenvironment where stem cells reside. In recent years, a variety of stromal cells in bone marrow have been reported to be the components of the HSC niche. These include osteoblastic lining cells, sinusoidal endothelium, CXCL12 abundant reticular cells and adipocytes. These stromal cells play important roles in maintaining HSCs and regulating the balance between HSC self-renewal and differentiation [5-11]. But, whether these stromal cells also influence the aging process of HSCs is largely unknown.

This recent paper describes a novel role of osteoblastic niche in influencing the aging of HSCs [1]. The authors used parabiotic (two surgically joined) mice to compare HSC phenotype in young and aged mice, as well as the influence of young and aged microenvironment on HSC aging. They used isochronic young (2 months old), isochronic old (18-22 months old), heterochronic young and old mice for the parabiotic pairs. Surface markers CD45.1 and CD45.2 were used to distinguish the parabiotic mice from each other. In the parabiotic system, HSCs were able to circulate between the surgically joined mice.

Using this system, the authors first asked: could aged HSCs be rejuvenated? Analyzing the number of HSCs and osteoblasts in different parabiotic pairs and checking HSC function by transplanting bone marrow cells derived from different parabiotic pairs into re- cipient mice, they found that HSCs from isochronic old pairs exhibited increased myeloid biased lineage potential and fewer B lymphoid cells than HSCs from isochronic young pairs. The number of osteoblastic niche cells also increased in isochronic old pairs. Surprisingly, HSCs transplanted from the heterochronic old mice into recipient mice not only restored the normal number of HSCs and osteoblastic niche cells, but also reversed the balance between myeloid and lymphoid lineages. These observations suggest that aging phenotype of HSCs and osteoblastic niche cells could be reversed by exposure to a young environment.

The second question the authors asked: could the young environment directly or indirectly rescue aged HSCs? They co-cultured osteoblastic niche cells isolated from isochronic young, isochronic old, and heterochronic pairs respectively with young HSPCs in vitro and found that isochronic old osteoblastic niche cells could promote proliferation of HSCs derived from young mice; whereas the behavior of osteoblastic niche cells in heterochronic old mice was similar to isochronic young mice. When the young HSPCs that had been co-cultured with osteoblastic niche cells from isochronic old, isochronic young, and heterochronic mice for 36 hours respectively were transplanted into recipient mice, the donor HSCs derived from the isochronic old mice showed fewer leukocytes, more myeloid and 
fewer B cells in the peripheral blood than donor cells from isochronic young mice. In contrast, the donor HSCs derived from heterochronic old mice had similar numbers of leukocytes, myeloid, and $\mathrm{B}$ lymphoid cells as the donor cells from isochronic young mice. This observation indicates that osteoblastic niche cells could directly influence HSC aging.

When young osteoblastic niche cells were treated with serum from old mice for one day and then co-cultured with young HSPCs, intriguingly the number of HSPCs increased. In contrast, when old osteoblastic niche cells were treated with young serum, the number of HSPCs decreased in the same experimental setting. These observations suggest that the serum from young mice could directly rejuvenate the osteoblastic niche cells in the old mice, which in turn rejuvenated HSCs.

The third question the authors asked: what is the molecular mechanism by which circulating signals influence the osteoblastic niche, which in turn rejuvenates aged HSCs? They found that local insulin-like growth factor-1 (IGF-1) plays an important role in regulating the function of osteoblastic niche cells. When old osteoblastic niche cells were treated with anti-IGF neutralizing antibody, or when young osteoblastic niche cells were treated with old serum and anti-IGF neutralizing antibody in vitro and then co-cultured with young
HSPCs, the expansion of HSCs was inhibited. When anti-IGF neutralizing antibody was injected into the tibia of old mice and then old osteoblastic niche cells were isolated and co-cultured with HSPCs, the anti-IGF neutralizing antibody restored the normal number of HSCs. This finding indicates that IGF-1 signal impaired the regulatory function of the aged osteoblastic niche and resulted in age-associated hematopoietic phenotype.

Based on these observations, the authors propose a new model that aged circulating soluble factors affect the level of IGF-1 in the local osteoblastic niche, which in turn contributes to age-related dysfunction of HSCs, including biased differentiation of HSCs to myeloid over B lineages. Heterochronic circulating partially neutralizes IGF-1 signaling in the osteoblastic niche, resulting in rejuvenation of aged HSCs. This elegant research opens a new avenue for clinical therapy, such as targeting the circulation factors in blood to restore the function of niche cells and HSCs.

\section{References}

1 Mayack SR, Shadrach JL, Kim FS, Wagers AJ. Systemic signals regulate ageing and rejuvenation of blood stem cell niches. Nature 2010; 463:495-500.

2 Rossi DJ, Jamieson CH, Weissman IL. Stems cells and the pathways to aging and cancer. Cell 2008; 132:681-696.

3 Chambers SM, Shaw CA, Gatza C,
Fisk CJ, Donehower LA, Goodell MA. Aging hematopoietic stem cells decline in function and exhibit epigenetic dysregulation. PLoS Biol 2007; 5:e201.

4 Kohler A, Schmithorst V, Filippi M-D, et al. Altered cellular dynamics and endosteal location of aged early hematopoietic progenitor cells revealed by time-lapse intravital imaging in long bones. Blood 2009; 114:290-298.

5 Schofield R. The relationship between the spleen colony-forming cell and the haemopoietic stem cell. Blood Cells 1978; 4:7-25.

6 Zhang J, Niu C, Ye L, et al. Identification of the haematopoietic stem cell niche and control of the niche size. Nature 2003; 425:836-841.

7 Calvi LM, Adams GB, Weibrecht KW, et al. Osteoblastic cells regulate the haematopoietic stem cell niche. Nature 2003; 425:841-846.

8 Kiel MJ, Yilmaz OH, Iwashita T, Terhorst C, Morrison SJ. SLAM family receptors distinguish hematopoietic stem and progenitor cells and reveal endothelial niches for stem cells. Cell 2005; 121:1109-1121.

9 Sugiyama T, Kohara H, Noda M, Nagasawa T. Maintenance of the hematopoietic stem cell pool by CXCL12-CXCR4 chemokine signaling in bone marrow stromal cell niches. Immunity 2006; 25:977-988.

10 Yin T, Li L. The stem cell niches in bone. $J$ Clin Invest 2006; 116:1195-1201.

11 Naveiras O, Nardi V, Wenzel PL, Hauschka PV, Fahey F, Daley GQ. Bone-marrow adipocytes as negative regulators of the haematopoietic microenvironment. Nature 2009; 460:259263. 